\title{
Leistungserfassung im OP
}

\author{
Kerstin F. Schlegel, Peter C. Strohm, Regina Dries, Rainer Burkhardt, \\ Norbert P. Südkamp
}

\section{Zusammenfassung}

In einem modernen Krankenhaus ist eine EDV-gestützte Leistungserfassung, insbesondere im Operationssaal, nicht mehr wegzudenken. $\mathrm{Zu}$ einer vollständigen Leistungserfassung zählt nicht nur die patientenbezogene Dokumentation der medizinischen Daten und Leistungen, sondern auch die Dokumentation aller abrechnungsrelevanten Informationen. Durch den Gesetzgeber sind diesbezüglich Vorgaben gemacht, welche sich im Gesundheits- strukturgesetz und im Sozialgesetzbuch V verankert finden. Eine vollständige Dokumentation muss sowohl von pflegerischer, als auch ärztlicher Seite durchgeführt werden. Sie sollte mit möglichst geringem Aufwand und einfach durchzuführen sein. Auch im Hinblick auf abnehmende Ressourcen, drohenden Stellenabbau und zunehmende Arbeitsbelastung in allen Bereichen ist eine umfassende und genaue Leistungserfassung wichtig und notwendig.

\section{Einleitung}

Als Konsequenz des Gesundheitsstrukturgesetzes vom 1. Januar 1996 sind Krankenhäuser aufgefordert, medizinische Leistungen zeitnah zu dokumentieren und einer vergleichenden Prüfung zugänglich zu machen.

Die Verpflichtung zur patientenbezogenen Übermittlung diagnose- und therapierelevanter Daten unter Nutzung der elektronischen Datenverarbeitung ( $§ 301$ Sozialgesetzbuch V) soll Transparenz in die Qualität medizinischer Leistungen und deren Kosten bringen.

Um diesen Anforderungen gerecht zu werden, ist eine Leistungserfassung in allen Bereichen, speziell im Operationsbereich (OP) als Leistungsstelle, kaum zu umgehen, ja sogar Voraussetzung [1,2,6].

Leistungserfassung bedeutet detaillierte patientenbezogene Dokumentation sowohl medizinischer als auch abrechnungsrelevanter Daten.

OP-JOURNAL 2005; 21: 74-78
Weiterhin muss natürlich die Leistung des beteiligten Personals dokumentiert werden. Über die Erfüllung der vom Gesetzgeber gestellten Anforderungen hinaus ermöglicht eine gute Dokumentation einen schnellen Zugriff auf alle Daten und deren statistische Auswertung, sofern diese für das interne Qualitätsmanagement, für Wissenschaft und Forschung oder für weitere Therapiemaßnahmen benötigt werden. Auch im Falle forensischer Fragestellungen ist durch eine Leistungserfassung ein schneller Zugriff möglich und eine lückenlose Dokumentation gewährleistet [6].

Auf dem freien Markt werden die verschiedensten Klinikinformationssysteme und OP-Dokumentationsprogramme zur Verschlüsselung nach der International Classification of Diseases (ICD) - aktuell der 10. überarbeiteten Fassung ICD-10 (2005) - und dem Operationen- und Prozedurenschlüssel (OPS-301) angeboten. Im Auftrag des Grundsatzausschuss der Deutschen Gesellschaft für Unfallchirurgie (DGU) erfolgte im Jahr 2003 eine multizentrische Umfrage in deutschen unfallchirurgischen Kliniken bezüglich der verwendeten Kliniksoftware. 180 der 285 angeschriebenen Kliniken (63\%) nahmen an der Befragung teil. In 24\% der Kliniken wurde zu dem Zeitpunkt die Software von SAP verwendet. Ebenfalls 24\% nutzten das System von Siemens. Weiterhin kamen Klinikinformationssysteme von GWI/Orbis ${ }^{\circledR}$ (14\%), GAP (6\%), BOSS (5\%), Promt (3\%) und Sonstige (24\%) zum Einsatz (Abb.1). 88\% aller beteiligten Kliniken setzten ihre Software im OP und zur OP-Planung, 98\% ebenfalls zur ICDund OPS-Verschlüsselung ein.

Marktbeobachtungen und die Testung verschiedener Produkte haben jedoch gezeigt, dass ein solches System für ein Großklinikum nicht einfach „,von der Stange“ gekauft werden kann [3]. Daher wurde das hiesige Klinikrechenzentrum beauftragt, ein Klinikinformationssystem eigens für das Uniklinikum Freiburg zu entwickeln. Seit 1994 entstand das Planungs- und Dokumentationssystem PROMetheus, welches eng an das zentrale Patienten-Daten-Verwaltungssystem (PDVFR) gekoppelt ist und einen Datenabgleich der Patientendaten und die Übermittlung derselben an die Krankenkassen gewährleistet [4]. Durch engen Kontakt mit den Anwendern ist es möglich, dieses Programm sowohl schnell und individuell als auch abteilungsspezifisch weiterzuentwickeln. Integriert in das Programm findet sich neben der vollständigen, elektronischen Patientenakte mit allen Befunden (Labor, Mikrobiologie,

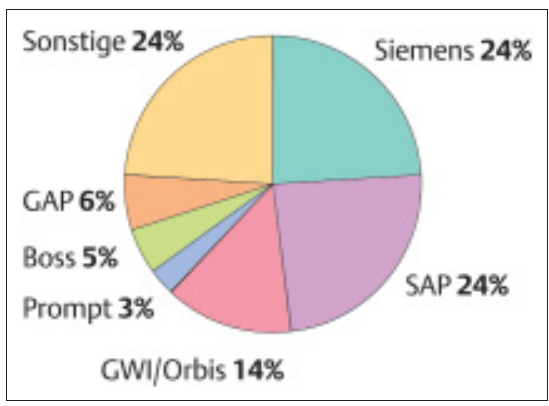

Abb.1 Verwendete Klinikinformationssysteme nach Auswertung von 180 der 285 unfallchirurgischen Kliniken Deutschlands, Erhebung des Grundsatzausschusses der DGU 2003. 
Radiologie mit Bildern, Pathologie usw.), Dokumentenverwaltung mit Arztbriefen, OP-Berichten usw. auch die Planung der Funktionsbereiche wie Ambulanz-Planung, Bettenplanung sowie das Modul der OP-Planung und OP-Dokumentation [3].

Im Folgenden soll exemplarisch die in den Operationssälen des Departments für Orthopädie und Traumatologie des Uniklinikums Freiburg durchgeführte Leistungserfassung und OP-Dokumentation vorgestellt und erläutert werden.

\section{Strukturelle Voraussetzungen}

Sowohl in jedem Arztzimmer, auf den Stationen, in der Ambulanz und in den Sekretariaten, als auch in jedem der Operationssäle und in der OP-Leitstelle findet sich ein EDV-Arbeitsplatz mit Zugang für das Klinikinformationssystem PROMetheus. Durch den Systemadministrator erhält jeder Mitarbeiter eine Zugangsberechtigung mit Benutzernamen und Passwort. Je nach Arbeitsplatz erfolgt eine spezifische Konfiguration der Programm-Module. Darüber hinaus können für die einzelnen Abteilungen des Universitätsklinikums spezielle Dokumentationsmasken zur Verfügung gestellt werden.

Alle an dem Operationsvorgang beteiligten Fachbereiche haben Zugriff auf die OP-Planung und können die wichtigen Informationen eingeben oder entnehmen.

\section{Programmbeschreibung}

An den Arbeitsplätzen in den Operationssälen öffnet sich nach Einloggen in das System die zentrale Bedienmaske für die OP-Planung. In einer Stundenplandarstellung werden für den ausgewählten Tag alle patientenbezogenen Termine in den einzelnen OP-Sälen angezeigt (Abb. 2). Durch Doppelklick des gewünschten Patienten öffnet sich die individuelle OP-Dokumentation mit der Anmeldung als erste Maske (Abb.3). Von dort lässt sich auf weitere Karteikartenreiter zugreifen, die den Benutzer durch die gesamte Leistungserfassung führen.

Die zu erhebenden Daten sind in ärztliche und pflegerische Teile gegliedert. Bis auf wenige Ausnahmen erfolgt die Bearbeitung der OP-Anmeldung und des OP-Kurzberichtes durch den Arzt. Die übrige Dokumentation der OP-Angaben erfolgt durch die Pflege.

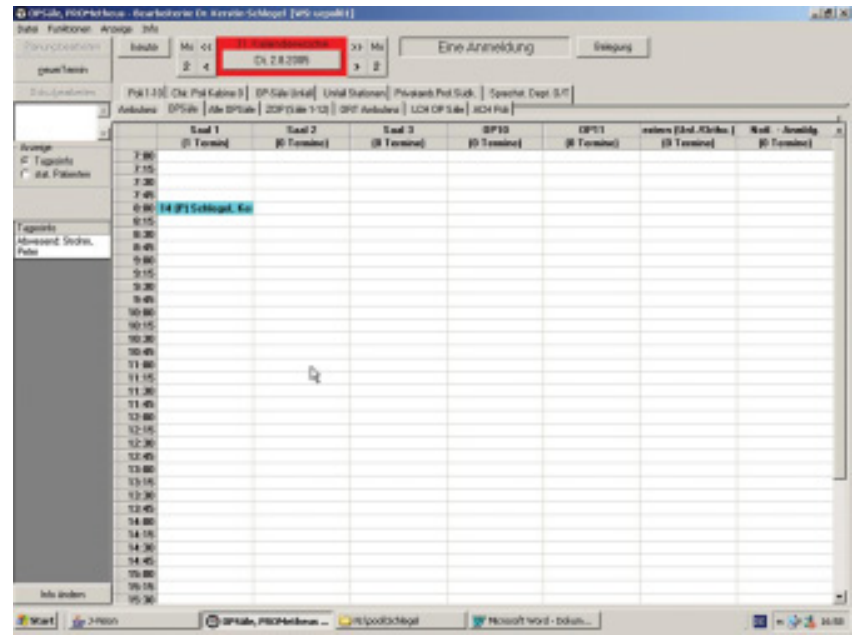

Abb. 2 Übersicht der OP-Säle in Stundenplandarstellung.

Nach der vollständigen Eingabe aller relevanten Daten muss entsprechend „Arzt fertig“ bzw. „Pfleger fertig“ angekreuzt werden. Wenn beides angekreuzt ist, wird die OP-Dokumentation nach vorheriger Warnung archiviert und kann anschließend nicht mehr verändert werden.

\section{Maske 1: OP-Anmeldung}

Auf der ersten Seite (Abb.3) finden sich zunächst die Patientendaten, wie sie bereits im PROMetheus erfasst sind, sowie die Station, von welcher der Patient kommt und auf die er postoperativ zurückverlegt werden soll. In den Infotext wird vom anmeldenden Arzt die präoperativ gestellte Diagnose und der geplante Eingriff als Freitext eingegeben, dieser erscheint ebenfalls in der zentralen Bedienmaske der OP-Planung. Besonderheiten wie Lagerung, spezielle Implantate oder relevante Informationen müssen eben- falls hier eingegeben werden. Weiterhin können die Namen des ärztlichen Personals eingesehen und bearbeitet werden. Für die abteilungsinterne Komplikationsstatistik ist ebenfalls ein Eingabefeld vorgesehen, welches ärztlicherseits ausgefüllt wird.

Nach beendeter Operation werden vom Operateur die abrechnungsrelevanten Diagnosen und Prozeduren nach den gängigen Thesauren wie ICD und OPS301 verschlüsselt und eingegeben, diese werden unmittelbar an das zentrale Patienten-Daten-Verwaltungssystem (PDV$F R)$ übertragen.

Diagnose- und Therapieverschlüsselung sind grundsätzlich ärztliche Aufgabe. In der Maske Anmeldung 2 bietet sich Platz für Bemerkungen der Anästhesiologie, die ebenfalls als Freitext eingegeben werden können (Abb.4).

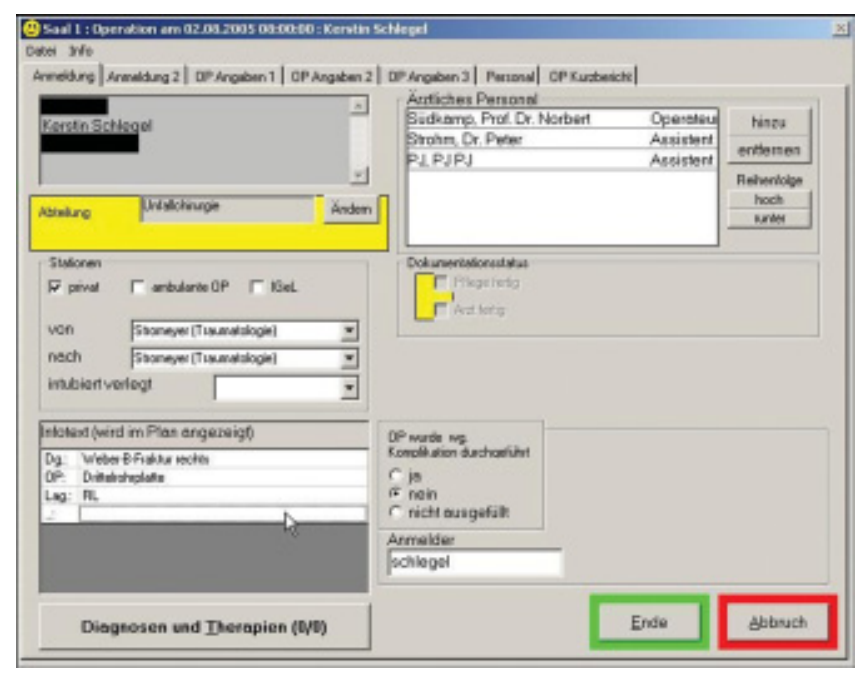

Abb. 3 Maske der OP-Anmeldung mit Zugriff auf weitere Karteikartenreiter im oberen Bildrand. Hier können bei der Anmeldung alle relevanten Informationen eingegeben werden und erscheinen so auch im OP-Plan. 


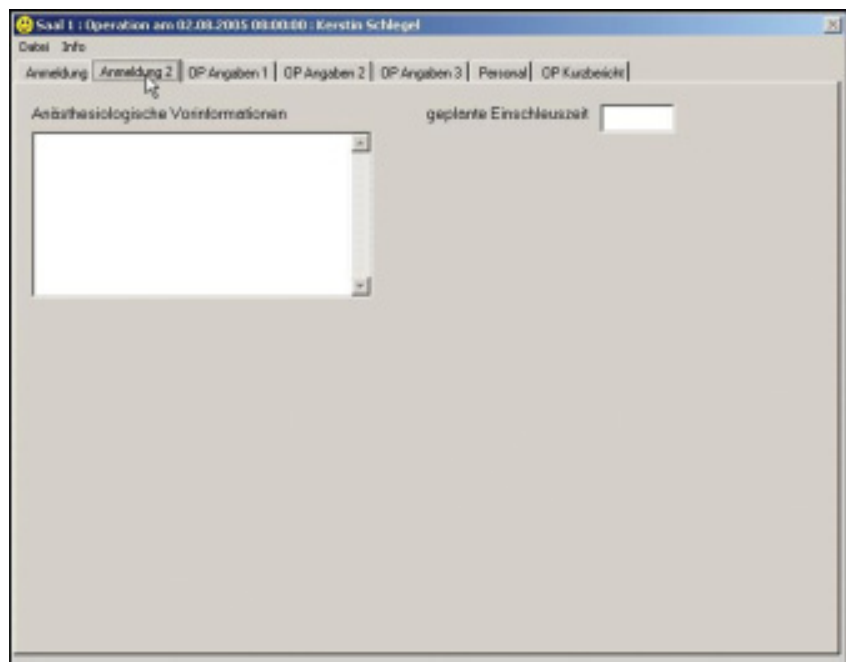

Abb. 4 OP-Anmeldung 2, Möglichkeit zur Eingabe von Vermerken der Abteilung für Anästhesie.

\section{Maske 2: OP-Angaben 1}

Nach Anklicken der Maske OP-Angaben 1 (Abb.5) finden sich Eingabefelder zur Dokumentation der Narkoseart, der Patientenlagerung und der zum Einsatz gekommenen Geräte wie z.B. Röntgen und Warm Touch. Weiterhin wird die Position der Neutralelektrode, sofern sie zum Einsatz kommt, und die verwendete Methode der Elektrokoagulation (mono- oder bipolar) belegt. Bei Anlage eines Blasenkatheters wird diese Maßnahme sowie die durchführende Pflegekraft dokumentiert. Wärmematten kommen, nachdem erst kürzlich sämtliche OP-Tische ungerüstet wurden, nicht mehr zum Einsatz. Im Eingabefeld Anmeldung wird erfasst, ob es sich um einen elektiven, dringlichen oder um einen Notfalleingriff handelt. Die meisten Angaben können aus Auswahllisten selektiert werden, so dass die Bedienung schnell und einfach erfolgt. Im Mittelpunkt dieser Maske fin- det sich jedoch die Erfassung der OP-Zeiten. Die Rüstzeiten umfassen die gesamte Vor- und Nachbereitungsphase der Operation. Sie beinhalten Tätigkeiten wie Umkleiden, Waschen und Händedesinfektion, die Vorbereitung des Instrumentariums und des Saals, Lagerung des Patienten, sowie Tätigkeiten wie die postoperative Patientenversorgung, das Verräumen des Instrumentariums, die Nachbereitung des Operationssaals und zuletzt die Dokumentation der Operation. Der Beginn der operativen Maßnahme wird als der Zeitpunkt definiert, zu dem die OP-Pflege bzw. der Assistenzarzt oder Operateur erstmals am Patienten arbeitet, in der Regel handelt es sich dabei um die ersten Maßnahmen der Lagerung. Eventuell entstandene Wartezeiten können nach Anklicken des Buttons auf einer separaten Seite belegt werden. Die Abrufzeit des Pflegepersonals während der Bereitschafts- und Rufdienstzeiten wird ebenfalls dokumentiert. Zuletzt findet

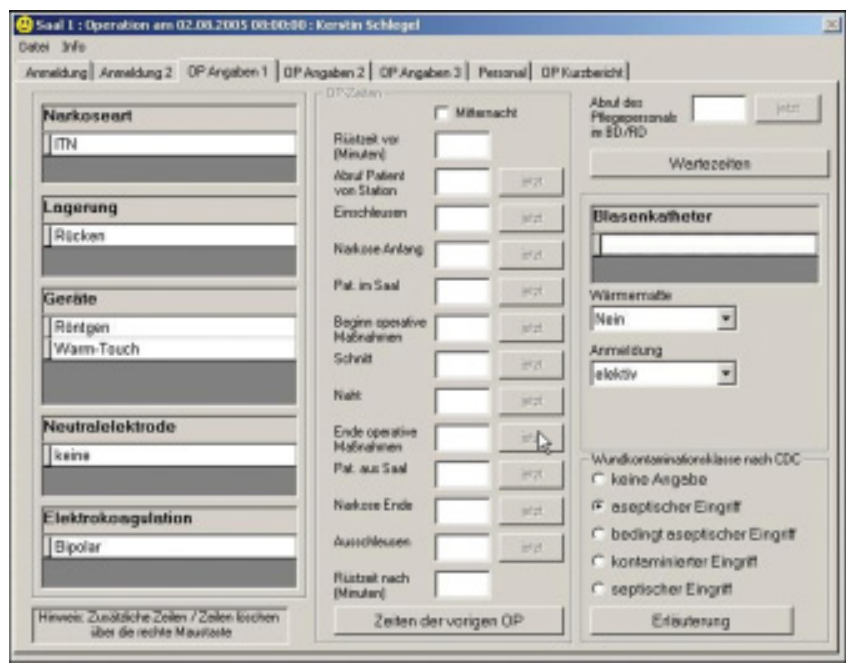

Abb. 5 OP-Angaben 1, Zeitdokumentation des OP-Ablaufs, Dokumentation von Lagerung, Geräten und Narkoseform. Hier muss auch die Wundkontaminationsklasse dokumentiert werden (unten rechts). sich Raum zur Erfassung der Wundkontaminationsklasse nach CDC (Centers for Disease Control and Prevention) durch Anklicken des entsprechenden Feldes durch den Arzt.

\section{Maske 3: OP-Angaben 2}

In dieser Maske (Abb.6) werden die verwendeten Implantate dokumentiert. Sowohl die Bestellnummer, Größe und Anzahl als auch die Chargennummer und der Hersteller werden erfasst. So kann beispielsweise bei späteren Komplikationen oder fraglichem Implantatversagen das verwendete Material einfach identifiziert und der Hersteller entsprechend informiert werden. Entnommene Präparate für die Mikrobiologie oder Pathologie werden ebenfalls erfasst. Weiterhin werden bei Anlage einer Blutsperre die Lokalisation und die Zeitspanne, in der sie zum Einsatz kommt, dokumentiert. Sofern während der Operation geröntgt wird, werden zuletzt die Anzahl der angefertigten Bilder mit Durchleuchtungsdauer, Lokalisation und die applizierte Gesamtdosis niedergelegt. Auch im Falle einer Gipsanlage oder Bronchoskopie während des operativen Eingriffs werden diese Maßnahmen dokumentiert.

\section{Maske 4: OP-Angaben 3}

In der letzten Maske der OP-Angaben (Abb.7) findet sich Raum zur Dokumentation verwendeter Flüssigkeiten, wie z.B. Spülflüssigkeiten, Haut- und Schleimhautdesinfektionsmittel, sowie vom Operateur lokal eingesetzter Medikamente, wie z.B. Lokalanästhetika. Weiterhin werden Bauchtücher, Streifen etc. mit Zählproben dokumentiert. Eingelegte Drainagen mit Anzahl, Lokalisation und Größe werden hier ebenfalls erfasst. Eine Thromboseprophylaxe wird selten während des operativen Eingriffs durchgeführt, sie erfolgt in der Regel bereits im Vorfeld auf Station. Besonderheiten oder Unregelmäßigkeiten (Arztwechsel, Instrumentier- oder Springerwechsel, Klimatechnikprobleme etc.) während der Operation werden in dem entsprechend markierten Eingabefeld hinterlegt.

\section{Maske 5: Personal}

Die Dokumentation des beteiligten Personals mit Funktion wie Instrumentierender, Springer, Gipser oder Lagerung erfolgt in dieser vorletzten Maske (Abb.8). Die einzelnen Personen können einer Auswahlliste entnommen und per 

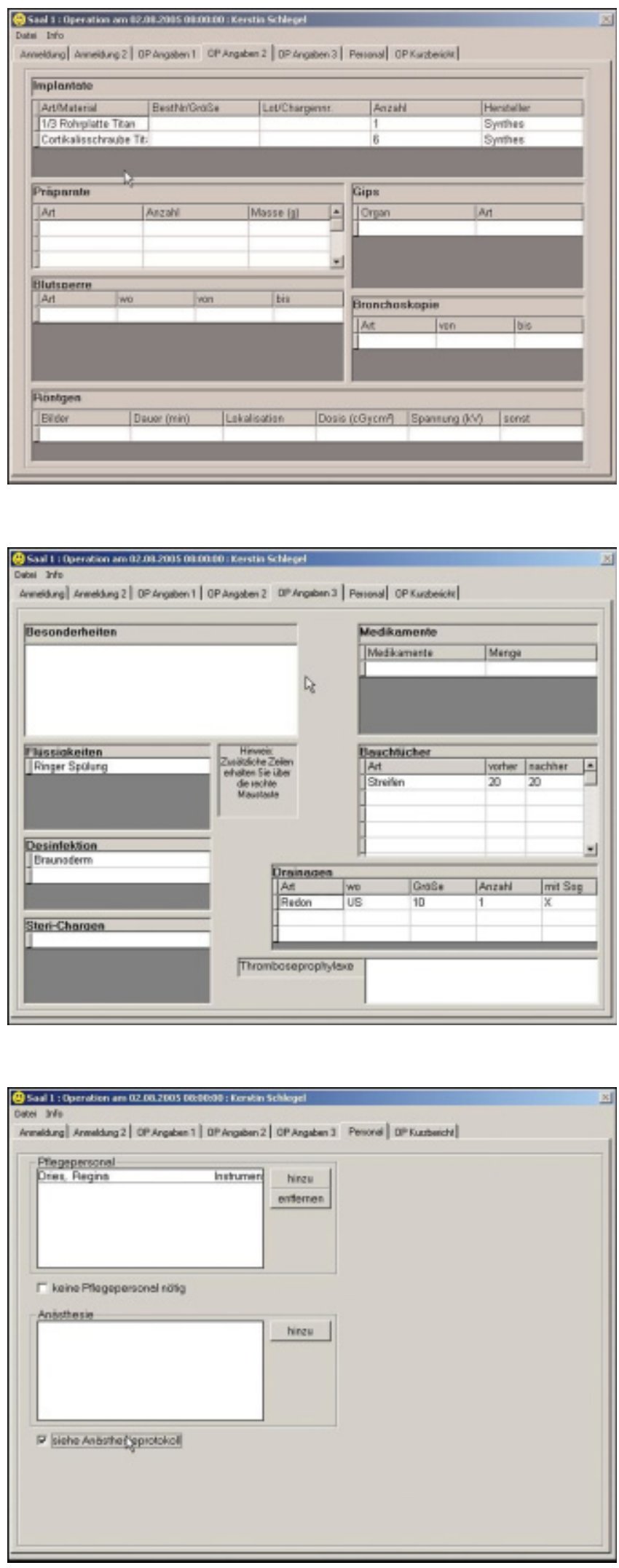

Abb. 6 OP-Angaben 2, Dokumentation von verwendeten Implantaten, Röntgen, Gips u.ä.

Abb. 7 OP-Angaben 3, Dokumentation von Medikamenten, Spülflüssigkeiten, Drainagen, Desinfektion usw.

Abb. 8 Dokumentation des Pflegeund Lagerungspersonals, Möglichkeit zur Dokumentation des Anästhesiepflegepersonals.
Mausklick eingegeben werden. Hier wird auch der für die Lagerung verantwortliche Arzt dokumentiert. Das Anästhesiepersonal wird in der Regel mit Verweis auf das Anästhesieprotokoll dokumentiert. Dieses wird zunächst nicht elektronisch erfasst, sondern handschriftlich geführt. In einem weiteren Arbeitsschritt werden dann die Protokolle über ein Belegleseverfahren in eine Datenbank eingespielt. Die hierfür verwendete Software heißt AnDok.

\section{Maske 5: OP-Kurzbericht}

Mithilfe der letzten Maske wird am Ende der Operation vom Operateur ein OPKurzbericht erstellt (Abb.9u.10). Nach Ausfüllen der Textbausteine wird dieser in dreifacher Ausführung ausgedruckt. Zwei Exemplare werden dem Pflegepersonal direkt bei Abholung des Patienten auf Station gegeben, so dass dokumentierte Anordnungen unmittelbar und ohne weitere Rücksprache mit dem Operateur durch Pflege und Physiotherapie durchgeführt werden können. Ein weiteres Exemplar dient dem Operateur zur Vorlage beim Diktieren des endgültigen Operationsberichts. Im OP-Kurzbericht werden Besonderheiten jeder Art während der Operation beschrieben sowie patientenspezifische Risiken, wie z.B. ein erhöhtes Risiko für Wundheilungsstörungen, Nachblutungen oder ein erhöhtes Infektionsrisiko, dokumentiert. Darüber hinaus erfolgt eine Einschätzung des Blutverlustes während der Operation. Weiterhin wird bei Applikation eines Antibiotikums während des Eingriffs bzw. die weitere Anordnung während der postoperativen Phase dokumentiert. Eingelegte Drainagen werden ebenfalls zur Information für die Stationen aufgeführt. In zwei weiteren Feldern bietet sich Platz zur Klassifikation der behandelten Frakturen nach AO (Arbeitsgemeinschaft für Osteosynthesefragen) [5] und zur Weichteilklassifikation nach Tscherne/Oestern [7]. In unserer Klinik wurde in Zusammenarbeit mit der Anästhesie und des Schmerzdienstes ein 3-Stufen-Schmerzschema, angelehnt an die Empfehlungen der WHO (World Health Organization), entwickelt. Durch Angabe der Schemastufe kann auf den Stationen ohne weitere Anordnung sofort mit der postoperativen Schmerztherapie begonnen werden. Durch ein einfaches Anklicken des entsprechenden Feldes erfolgt die Anordnung der postoperativen Thromboseprophylaxe und Lymphdrainage. Darüber hinaus können Anordnungen z.B. für die Physiotherapie bezüglich Ausmaß 

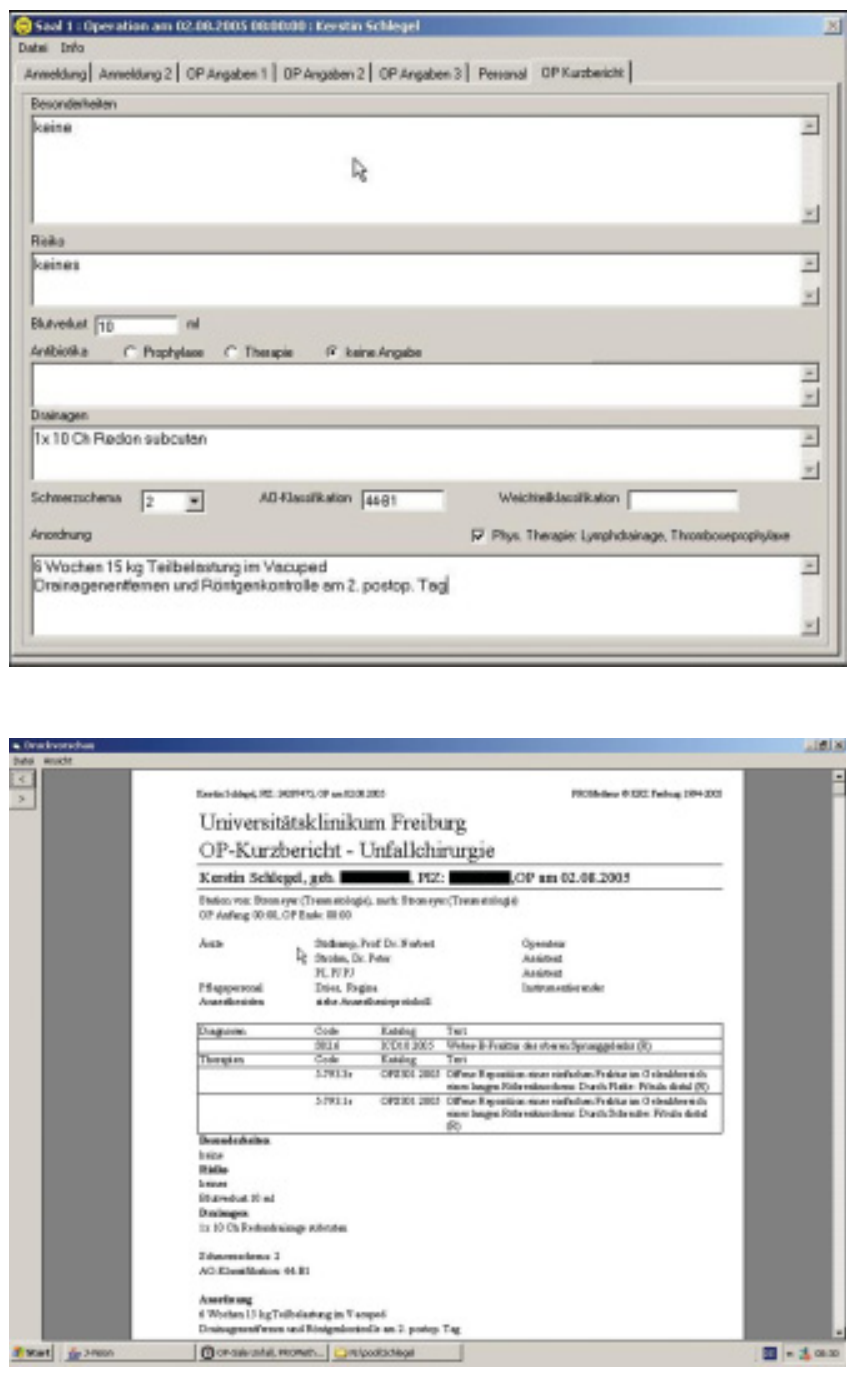

Abb.10 OP-Kurzbericht, welcher direkt zum Diktat, auf Station und zur Abteilung für Physiotherapie geht. der Beübung und Mobilisation im letzten Eingabefeld dokumentiert werden.

\section{Schlussfolgerung}

Am Beispiel der an unserer Klinik durchgeführten OP-Dokumentation sollen die funktionellen, inhaltlichen und technischen Anforderungen an eine Leistungserfassung verdeutlicht werden. Aber auch gesetzliche Vorgaben müssen erfüllt sein. In $\S 301$ SGB V ist festgelegt, dass bestimmte, patientenbezogene Angaben,
Abb. 9 Kurzbericht des Operateurs über OP-Verlauf und Nachbehandlung. und die Verfügbarkeit an allen Leistungsund Schnittstellen (OP, Ambulanz, Stationen etc.) von Interesse.

\section{Literatur}

1 von Eiff W. Krankenhaus-Management: Qualität und Wirtschaftlichkeit als Managementherausforderung des Arztes. Zentralbl Chir 1996; 121: 817-127

2 Fischer H, Gerhardt EP, Greulich A, Räpple T, Schneider E, Thiele G, Ulmer HU, DegenerHencke U. Management-Handbuch Krankenhaus. Economica, Heidelberg 61. Aktualisierung November 2004

3 http://krzinfo/service/dokumentationen/ anwendungen/prometheus/Prometheus.html (Intranet UKL-Freiburg)

4 http://www.uniklinik-freiburg.de/z/krz/ apdv/de/pub/

5 Müller ME, Nazarian S, Koch P, Schatzker J. The Comprehensive Classification of Fractures of the Long Bones. Springer-Verlag Berlin-Heidelberg-New York 1990

${ }^{6}$ Roetman B, Zumtobel V. Klinische Informationssysteme: Strategien zur Einführung. Dt Ärztebl 2001; 98: A892 - 894

7 Tscherne H, Oestern HJ. Die Klassifizierung des Weichteilschadens bei offenen und geschlossenen Frakturen. Unfallheilkunde 1982; 85: $111-115$

\section{Dr. med. Kerstin F. Schlegel}

Assistenzärztin

\section{Dr. med. Peter C. Strohm}

Oberarzt

Regina Dries

OP-Gruppenleitung

Univ.-Prof. Dr. med.

Norbert P. Südkamp

Ärztlicher Direktor

Universitätsklinikum Freiburg

Department für Orthopädie und

Traumatologie

Hugstetterstr. 55

D-79106 Freiburg

Eine OP-Dokumentation allein genügt also nicht, wenn eine reibungslose Übermittlung an die Krankenkassen durch das Klinikinformationssystem nicht gewährleistet ist.

Für den Benutzer ist letztlich eine einfache und übersichtliche Bedienbarkeit sowie eine kurze Reaktionszeit des Systems

\section{Rainer Burkhardt}

Informatiker

Universitätsklinikum Freiburg

Klinikrechenzentrum

Abt. Klinische Arbeitsplatzsysteme

Agnesenstr. 6-8

D-79106 Freiburg 